\title{
Deregulation of energetic metabolism in the clear cell renal cell carcinoma: A multiple pathway analysis based on microarray profiling
}

\author{
ANDREA SOLTYSOVA $^{1,2}$, JAN BREZA $^{3}$, MARTINA TAKACOVA ${ }^{6}$, JANA FERUSZOVA $^{1}$, \\ SONA HUDECOVA ${ }^{1}$, BARBORA NOVOTNA $^{4}$, EVA ROZBORILOVA $^{5}$, SILVIA PASTOREKOVA ${ }^{6}$, \\ LUDEVIT KADASI $^{1,2}$ and OLGA KRIZANOVA ${ }^{1,6}$
}

\begin{abstract}
${ }^{1}$ Center for Molecular Medicine, Slovak Academy of Sciences, Bratislava; ${ }^{2}$ Department of Molecular Biology, Faculty of Natural Sciences, Comenius University, Bratislava; ${ }^{3}$ Department of Urology, University Hospital, Bratislava, Slovakia; ${ }^{4}$ Department of Neonatology and Pediatric Intensive Care, University Clinic Carl Gustav Carus, Dresden, Germany; ${ }^{5}$ Clinic of Pneumology and Pthisiology, Jessenius Medical Faculty, Martin;

${ }^{6}$ Institute of Virology, Slovak Academy of Sciences, Bratislava, Slovakia
\end{abstract}

Received March 25, 2015; Accepted May 4, 2015

DOI: 10.3892/ijo.2015.3014

\begin{abstract}
Clear cell renal cell carcinoma (ccRCC) is the most frequent type of kidney cancer. In order to better understand the biology of ccRCC, we accomplished the gene profiling of fresh tissue specimens from 11 patients with the renal tumors (9 ccRCCs, 1 oncocytoma and 1 renal B-lymphoma), in which the tumor-related data were compared to the paired healthy kidney tissues from the same patients. All ccRCCs exhibited a considerably elevated transcription of the gene coding for carbonic anhydrase IX (CAIX). Moreover, the ccRCC tumors consistently displayed increased expression of genes
\end{abstract}

Correspondence to: Professor Olga Krizanova, Center for Molecular Medicine, Slovak Academy of Sciences, Vlarska 7, 83101 Bratislava, Slovakia

E-mail: olga.krizanova@savba.sk

Abbreviations: AE1, AE3, AE4, anionic $\mathrm{Cl}-/ \mathrm{HCO}_{3}$-exchangers; ALDOC, aldolase C; ATP5A1, ATP synthase, H+ transporting, mitochondrial F1 complex, $\alpha$ subunit 1; CAIX, carbonic anhydrase IX; ccRCC, clear cell renal cell carcinoma; COX1, COX2, COX3, cytochrome oxidase 1, 2, 3; Glut1, Glut2, Glut3, glucose transporter of type 1, 2, 3; FBP1, fructose-1,6-bisphosphatase 1; HIF-1 $\alpha$, HIF-2 $\alpha$, hypoxia inducible factor $1 \alpha, 2 \alpha$; HK2, hexokinase II; KLF4, Kruppel-like factor 4; LDHA, LDHB, lactate dehydrogenase A, B; MCT, monocarboxylic acid transporters; MPC1, MPC2, mitochondrial pyruvate carrier of type 1, 2; NBCe1, NBCn1, electrogenic sodium bicarbonate cotransporters 1 of type e, n; ND, NADH dehydrogenase; OXPHOS, oxidative phosphorylation; PFK, phosphofructokinase; PGAM, phosphoglycerate mutase; RCC, renal cell carcinoma; VEGF, vascular endothelial growth factor; VHL, von Hippel-Lindau tumor suppressor

Key words: clear cell renal cell carcinoma, carbonic anhydrase IX, glucose transporters, hexokinase II, lactate dehydrogenase A encoding the glycolytic pathway enzymes, e.g. hexokinase II (HK2) and lactate dehydrogenase A (LDHA) and a decreased expression of genes for the mitochondrial electron transport chain components, indicating an overall reprogramming of the energetic metabolism in this tumor type. This appears to be accompanied by altered expression of the genes of the $\mathrm{pH}$ regulating machinery, including ion and lactate transporters. Immunohistochemical staining of tumor tissue sections confirmed the increased expression of CAIX, HK2 and LDHA in ccRCC, validating the microarray data and supporting their potential as the energetic metabolism-related biomarkers of the ccRCC.

\section{Introduction}

Renal cell carcinoma (RCC) is the most common malignancy arising in the adult kidney. Clear cell renal cell carcinoma (ccRCC) represents the most frequent subtype (83\%) of the RCC (1). The most striking phenotypic feature of the ccRCC is its clear cell morphology, which has been linked to a lipid and glycogen accumulation. Early diagnosis of ccRCC is associated with a favorable prognosis (5-year survival rate, $\sim 85 \%$ ). Unfortunately, ccRCC is often asymptomatic, with $\sim 30 \%$ of patients diagnosed at the metastatic stage when the prospects for cure are dismal (5-year survival rate, 9\%) (2).

The measurement of biomarkers in blood or tissue specimens has become an integral component of translational cancer research, with applications to studies on cancer etiology, treatment and prognosis, including early cancer detection (3). Diagnosis of the ccRCC is not so unambiguous, since there are no established serum biomarkers for the accurate diagnosis of this type of tumors. Reliable diagnostic biomarkers are urgently required. Accurate classification is clinically important because kidney tumor subtypes are associated with different malignant potential, prognosis and optimal therapies (4). 
Many alterations in the normal cellular hemostasis and metabolism in ccRCC occur in response to a so-called pseudo hypoxia (the activation of hypoxia-response pathways under normal oxygen conditions). Clear cell RCC typically exhibits this phenomenon because of specific molecular alterations. This type of carcinoma is closely associated with inactivating mutations of the von Hippel-Lindau tumor suppressor gene that lead to stabilization of hypoxia inducible factors (HIF-1 $\alpha$ and HIF- $2 \alpha$ ) in both sporadic and familial forms. Unlike normal cells, cancer cells metabolize glucose mostly via glycolysis, even in the presence of sufficient oxygen (5). Malignant cells have at least 20- to 30-fold higher rate of glycolysis than normal cells. It is widely accepted that increased glycolytic potential is one of the hallmarks of cancer (6).

While the Warburg effect may be related to energy, it is also clear that accumulation of lactate maintains a presiding influence over the acidic pericellular $\mathrm{pH}(\mathrm{pHe})$ circumscribing aggressive tumors. In turn, the presence of lactate is known to trigger aggressive forms of malignancy, augment metastases, chemoresistance and correlate to low survival rates (7).

Since ccRCC is a glycolytic and lipogenic tumor, in the present study, we focused on an identification of differentially expressed genes coding for proteins that regulate $\mathrm{pH}$ and drive energetic metabolism in collection of tumors and patientmatched healthy kidney samples. We aimed to analyze the common alterations in the ccRCC's mRNA levels in energyproducing processes related to the clear cell renal carcinoma and confirm the important changes observed on mRNA levels by immunohistochemistry.

\section{Materials and methods}

Patient information and tumor samples. Tumors from kidney of 11 patients ( 7 males, 4 females) with an average age of $62.2 \pm 2.5$ years were tested on changes in the gene expression profile of the energy-producing metabolism. Part of the unaffected kidney from the same patient was taken as a corresponding healthy control for the gene profiling. All samples were stored in RNA later. Of these patients, 9 patients were suffering from clear cell renal cell carcinoma, 1 patient suffered from renal oncocytoma and 1 from renal cell B-lymphoma. From ccRCCs, 1 belongs to the nuclear grade I, 1 was nuclear grade III and 1 was nuclear grade IV. All others belong to the nuclear grade II. The ethics committee of the IMPG SAS approved the present study and verbal consent was obtained from these participants prior to surgery.

$R N A$ isolation. Tumor and healthy tissues $1-1.5 \mathrm{~cm}^{2}$ in size were removed from RNA later, cut into smaller pieces and frozen in liquid nitrogen for $24 \mathrm{~h}$. Tissue was mechanically disrupted using mortar and pestle in liquid nitrogen. Total RNA was extracted from homogenized tissues using GeneJET ${ }^{\text {TM }}$ RNA Purification kit (Thermo Fisher Scientific, Waltham, MA, USA) according to the manufacturer's instructions. RNA quality was evaluated using Experion automated electrophoresis system for RNA analysis (Bio-Rad Laboratories) and RNA quantity was measured using NanoDrop ND 2000 (NanoDrop Technologies, LLC, Wilmington, DE, USA). Total RNA degradation/quality was determined based on $18 \mathrm{~S}$ and
$28 \mathrm{~S}$ ratio. RNA samples, where RQI number (calculated based on $18 \mathrm{~S} / 28 \mathrm{~S}$ ratio) from both, healthy and tumor tissues from the same patient was above 7.5, were selected for gene expression analysis.

Microarray assays. Total RNA (500 ng) was transcribed into cDNA, both strands of cDNA were synthesized using (dT) T7-primer. Subsequently labeling reaction was performed using Cy3-dCTP (healthy tissue samples) and Cy5-dCTP (tumor samples) to obtained cRNA. For this purpose, Quick Amp Labeling kit (Agilent Technologies, Santa Clara, CA, USA) was used. After labeling, samples were purified using GeneJE $^{\mathrm{TM}}$ RNA Purification kit (Thermo Fisher Scientific) to remove non-incorporated nucleotides. Yield of amplification process and specific activity were determined and only samples with specific activity above 8 proceeded to the hybridization step. A total of $300 \mathrm{ng}$ of appropriate labeled healthy tissue samples and tumor samples were mixed together and cRNA were fragmented by incubation for $30 \mathrm{~min}$ at $60^{\circ} \mathrm{C}$ using components from Gene Expression Hybridization kit (Agilent Technologies). Samples were immediately applied onto SurePrint G3 Human Gene Expression 8x60K v2 Microarray Slide (Agilent Technologies) and hybridized for $17 \mathrm{~h}$ at $65^{\circ} \mathrm{C}$ by rotating slide at $10 \mathrm{rpm}$ in Hybridization Oven (Agilent Technologies). After hybridization two wash steps were performed (Gene Expression Wash Buffer kit; Agilent Technologies) and slide was scanned at $2 \mu \mathrm{m}$ using NimbleGen MS 200 microarray scanner.

Image and data analysis. TIFF multiscan images from NimbleGen MS 200 scanner were converted using Feature Extraction software 11.5 (Agilent Technologies), the image processing was performed, and acquired files with spot intensities for every microarray field (corresponding to one patient) were imported into GeneSpring 12.6 GX software to analyze gene expression. Significant differences (fold of expression change $\geq 2.0$ ) in gene expression were evaluated for each patient separately (non-averaged) as well as averaged from all patients. Pathway analysis was performed to revealed molecular pathways significantly altered in our experiment $(\mathrm{P} \leq 0.05)$. Cluster analysis was performed using GeneSpring 12.6 GX for a selected set of genes.

Immunohistochemistry. Dissected tissues were embedded in paraffin according to the standard histological procedure. Sections $(4 \mu \mathrm{m})$ were placed on polylysine-coated slides, dewaxed and rehydrated. For CA IX detection, the immunostaining procedure was performed on an automated immunostainer (Dako Autostainer) using the DakoCytomation EnVision $^{\circledR}+$ System-HRP (DAB) for use with mouse primary antibodies according to the manufacturer's instructions: a) peroxidase and protein block (10 min each); b) incubation for $1 \mathrm{~h}$ with primary antibody (M75 hybridoma medium diluted 1:100 in antibody diluent) or PBS (negative control); c) incubation for 30 min with secondary antibody. Staining was visualized with DAB solution for 1 min with 3,3'-diaminobenzidine as a chromogenic substrate. The slides were washed in PBS with $0.1 \%$ Tween-20 for $10 \mathrm{~min}$ after step a, twice for 10 min after steps $b$ and $c$, and three times in distilled water after visualization with DAB. 
Table I. Significantly altered pathways of energetic metabolism.

\begin{tabular}{lll}
\hline Pathway & P-value & Altered genes
\end{tabular}

Glycolysis and gluconeogenesis

$2.5045212 \mathrm{E}-8$

Transport of glucose and other sugars, 1.3671912E-7 metal ions and amine compounds

Calcium regulation

Transport of inorganic cations-anions and amino acids-oligopeptides

3.492807E-6

1.0519273E-6

3.

Oxidative stress
Pyruvate metabolism and citric acid
(TCA) cycle

Monoamine transport

Metabolism of carbohydrates

\author{
3.241884E-4 MAOA, GPX3, NQO1, UGT1A6, CAT, TXNRD2, MT1X, FOS, \\ SOD2, SOD3, XDH, NOX4 \\ 4.0590137E-4 L2HGDH, D2HGDH, ADHFE1, aconitase 2 mitochondrial
}

9.3396695E-4 AMPH, PVRL2, SLC6A2, ITGB3, CDC25C, TDO2, AGT, SLC6A3, UNC13B, ADORA2A, IL1B, SLC6A4

0.024873339 PCK2, SLC25A10, G6PC, phosphoenolpyruvate carboxykinase 1 (soluble), trehalase, galactokinase
For HK2 and LDHA immunostaining, antigen retrieval was carried out with citrate buffer, $\mathrm{pH} 6.0$, for $5 \mathrm{~min}$ at $125^{\circ} \mathrm{C}$ using Pascal pressure chamber (Dako). Deparaffinized sections were stained with DakoCytomation EnVision ${ }^{\circledR}+$ System-HRP (DAB) for use with rabbit primary antibodies. Primary antibody specific for HXII (1:50; Cell Signaling Technology, Inc., Beverly, MA, USA) and LDHA (1:400; Cell Signaling Technology) was diluted in antibody diluent and incubated overnight at $4^{\circ} \mathrm{C}$. Staining was visualized with DAB solution.

All incubations and washings were carried out at room temperature. Finally, the sections were counterstained with Mayer's hematoxylin, washed for $5 \mathrm{~min}$ and were mounted in Aquamont (Merck, Darmstadt, Germany). The stained sections were examined with Leica DM4500 B microscope and photographed with Leica DFC480 camera.

\section{Results and Discussion}

The microarray analysis revealed significant changes in a variety of metabolism-related pathways in tumors vs. healthy kidney tissues, thus, suggesting a complex rearrangement of metabolic processes during tumor development. In the present study, we focused primarily on the glucose metabolism and its modulation. Glycolytic pathway belongs to the most modified ones in tumor ccRCC tissue, compared to the normal kidney tissue $\left(\mathrm{P}=2.5 \times 10^{-8}\right.$; Table I). Also, expression of the genes participating in the transport of glucose and other sugars, metal ions and amine compounds was significantly changed $\left(\mathrm{P}=1.4 \times 10^{-7}\right.$; Table $\left.\mathrm{I}\right)$ as described in more detail further below.

Hypoxia and $p H$. Uncontrolled cell proliferation within a developing tumor often outstrips its blood supply. Consequently, oxygen availability declines, but this physiological constrain is not sufficient to deprive cells of glucose. Prolonged hypoxic state leads to the stabilization of the hypoxia-inducible transcription factor 1 (HIF-1 $\alpha$ ), which helps cells to adapt to stressful environment by transactivating a broad spectrum of hypoxia-related proteins $(8,9)$. The major molecular event leading to the development of ccRCC is an inactivation or loss of the VHL gene (10-12). VHL protein is part of the E3-ubiquitin ligase complex that binds to the hypoxia-inducible factor subunit $\alpha$ (HIF- $\alpha$ ) under normoxic conditions and directs it to proteasomal degradation. Increased levels of the 

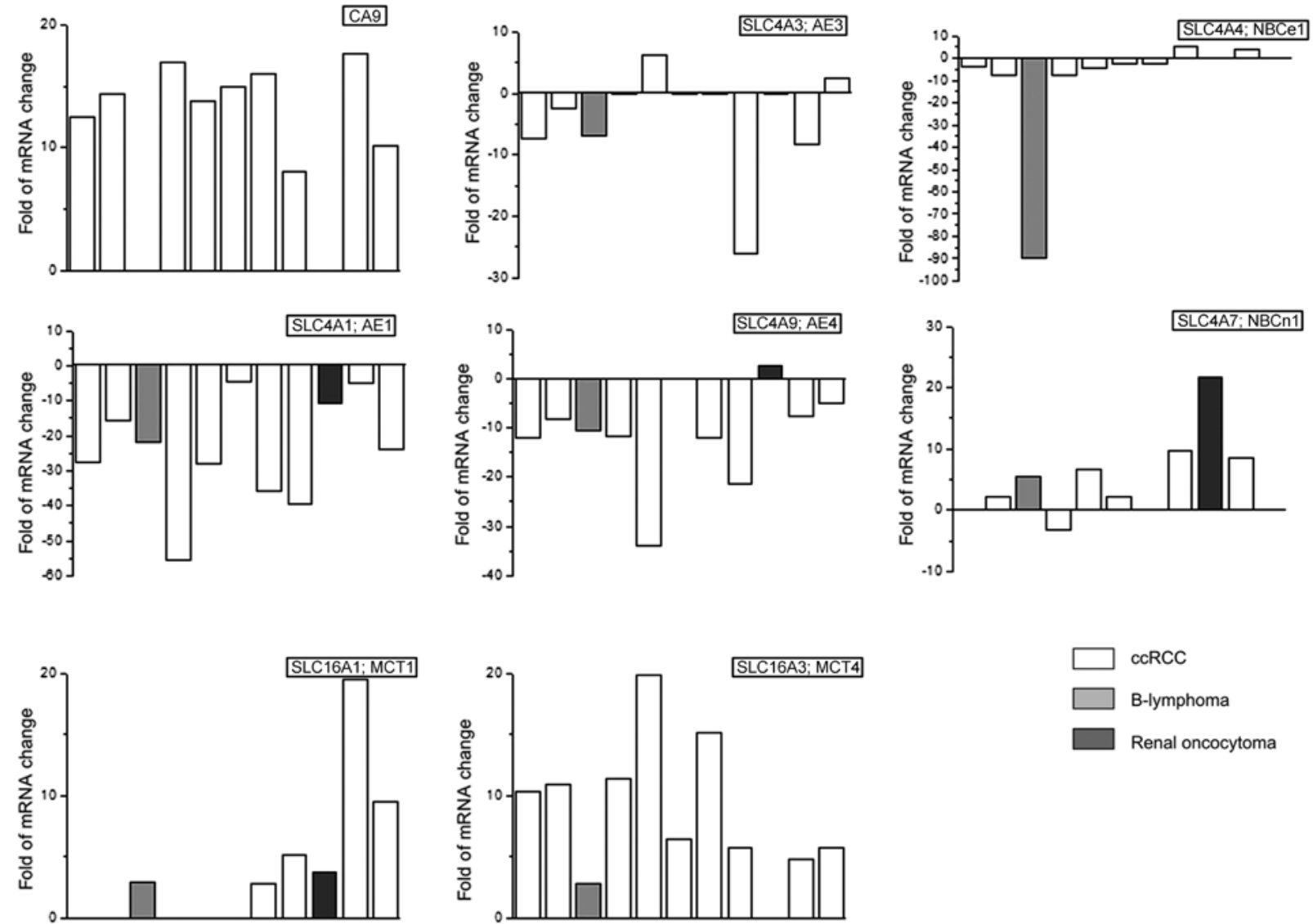

Figure 1. Expression profiles of $\mathrm{pH}$ regulators. Changes in expression of genes coding for CAIX, anion exchangers and bicarbonate anion transporters in ccRCC (empty columns), renal oncocytoma (dark gray column) and B-lymphoma (light gray columns).

HIF-1 resulting in upregulation of its target proteins equip ccRCC cells with a specific set of enzymatic machinery that favors aerobic glycolysis over oxidative phosphorylation (13). These factors support a shift toward a more glycolytic metabolism by stabilization of the HIF1 $\alpha$ and inhibition of prolyl hydroxylases. In order to distinguish, whether tumors of our patients were hypoxic and/or pseudohypoxic, we evaluated changes in the marker of hypoxia carbonic anhydrase IX (CA IX) (14). Expression of the CA IX coding gene was increased in 9 of 11 patients and the average increase was 12.6 \pm 1.3 -fold (Fig. 1). The 2 patients with no increase in CA IX expression suffered from renal oncocytoma (dark gray column) and B-lymphoma (light gray column), respectively. Moreover, immunohistochemistry proved increased CAIX staining in ccRCC tumor and no signal in renal oncocytoma and B-lymphoma (Fig. 3). Increased transcription of the gene coding for CA IX in ccRCC tumors would suggest that HIF1 $\alpha$ is stabilized and active. Also, this observation is in a clear agreement with the work of many other authors who suggested that CA IX is one of the best available markers for the clear cell RCC $(15,16)$.

It is well known that acidification of the tumor microenvironment often develops due to hypoxia-triggered oncogenic metabolism, which leads to the extensive production of lactate, protons and carbon dioxide. Mechanisms of $\mathrm{pH}$ regulation in tumor cells are very complex and intertwined with other cancer-related processes (17). Acidosis in tumor microenvironment is compensated by the alkalization of an intracellular
$\mathrm{pH}$ in cancer cells through the lactate and proton export and the bicarbonate import (17). CA IX is a catalytic component of the bicarbonate import arm, in which it cooperates with bicarbonate transporters (mainly with sodium-bicarbonate cotransporter NBCe1) and regulates $\mathrm{pH}$ in response to hypoxia or during cell migration-invasion $(18,19)$. Notably, ccRCC tumors displayed considerable decrease in expression of the genes encoding $\mathrm{Cl}^{-} / \mathrm{HCO}_{3}{ }^{-}$anion exchangers AE1, AE3 and $\mathrm{AE} 4$, which are considered acid loaders (the most pronounced decrease was observed in AEl that is normally expressed in intercalated cells of the renal collecting ducts (20). This is probably because cancer cells prefer acid extrusion to acid loading in order to preserve the accumulation of an intracellular acid). No significant change was found in the expression of AE2, in contrast to findings of Karumanchi et al (21), who proposed AE2 as potential VHL target. Furthermore, sodium-bicarbonate co-transporters revealed less marked changes. Expression of NBCe1 was mostly decreased (and this is possibly compensated by the strongly elevated CA IX), while levels of NBCe1 and NBCn1 were increased (the latter being previously associated with breast cancer) (Fig. 1). These data indicate existence of complicated crosstalk between the components of the $\mathrm{pH}$-regulating bicarbonate transport machinery, which is clearly deregulated in ccRCC.

Besides bicarbonate import, intracellular and extracellular $\mathrm{pH}$ is dependent on the lactate and proton export. Lactate is transported from the cells by monocarboxylic acid transporters (MCT; SLC16A), transmembrane proteins, which can trans- 

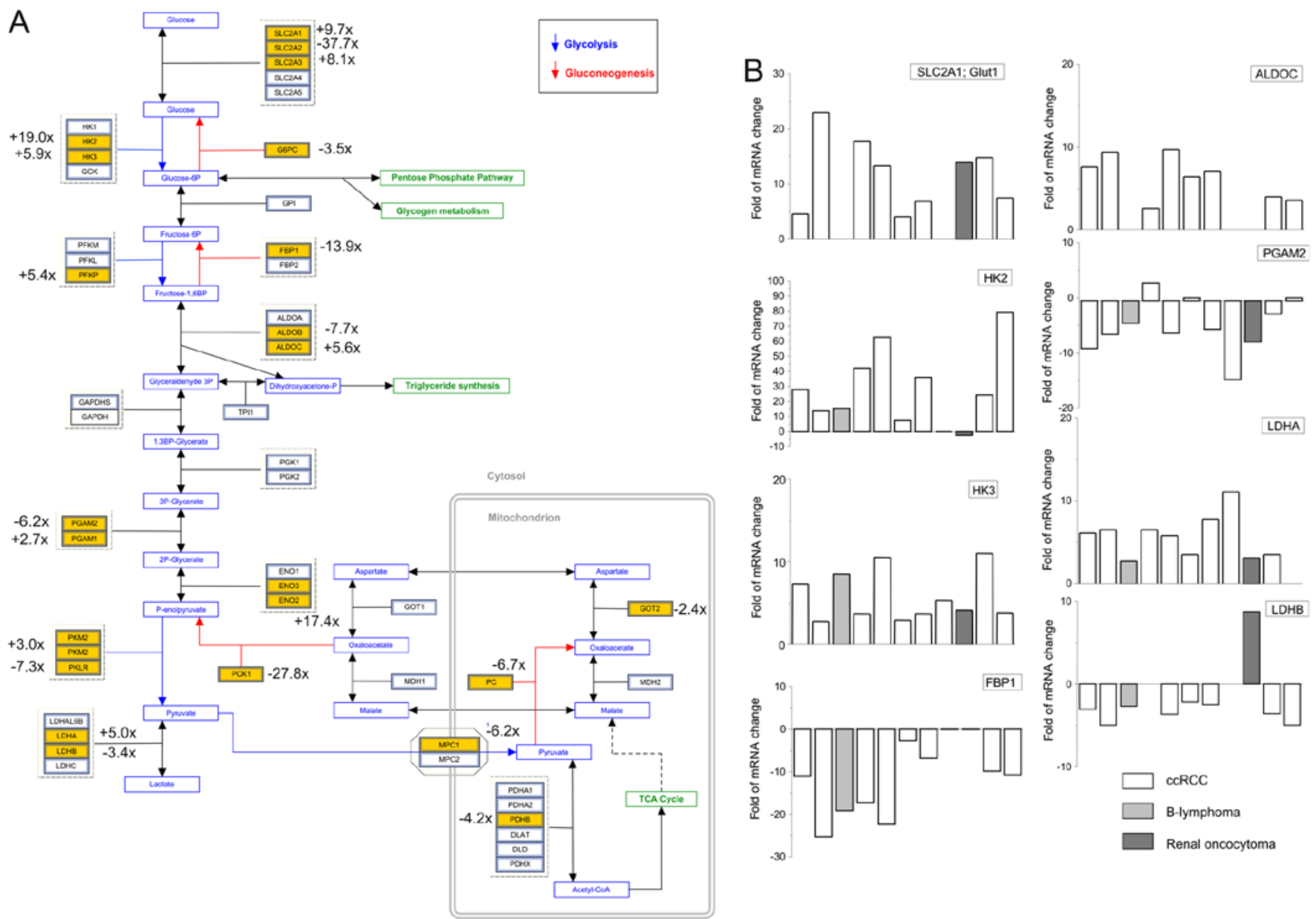

Figure 2. Glycolysis and gluconeogenesis in RCCs. (A) Genes coding for the enzymes of the glycolytic and gluconeogenetic pathways are shown in yellow, numbers indicate increase (+) or decrease (-) of their expression levels in tumors compared to the corresponding healthy kidney tissue. (B) Changes in expression of the genes encoding Glut1, HK2, HK3, FBP1, ALDOC, PGAM2, LDHA and LDHB in individual patients with ccRCC (empty columns), B-lymphoma (light gray column) and renal oncocytoma (dark gray column).

port lactate anion across the plasma membrane of tumor cells in association with proton (22). MCT1 (SLC16A1) and MCT4 (SLC16A3) are two isoforms most relevant for cancer physiology. From our cohort, all patients with diagnosed ccRCCs have increased expression of the MCT4 (10.0 \pm 1.7 -fold), but only 4 of them possess increased MCT1 (Fig. 1). Expression of the MCT4 was observed in many tumors, e.g. triple-negative breast (23), prostate cancer (24) and is always associated with poor prognosis.

Glucose transporters. HIF1 $\alpha$ is known to regulate expression of several enzymes of the glycolytic pathway. To increase the glucose uptake as a way to compensate low yield of ATP from glycolysis, HIF1 $\alpha$ promotes the overexpression of the glucose transporter of type 1 (Glut1; SLC2A1) and type 3 (Glut3; SLC2A3). In ccRCCs we observed increase in Glut1 in 8 patients (12.7 \pm 2.2 -times; Fig. 2) and no change in 1 patient. The Glut1 mRNA was also upregulated in the patient with the renal oncocytoma (Fig. 2, dark gray column) but no change was observed in the patient suffering from cell lymphoma. The Glut3 mRNA (SLC2A3) was also increased in ccRCCs in 7 patients (fold of increase $8.0 \pm 1.5$; Fig. 2); four tumors displayed no change compared to healthy tissues. Both of these transporters are associated with the tumorigenesis, however, the mechanism, as to which transporter is preferred, and why, is not known. Nevertheless, overexpression of the Glut1 and Glut3 in different tumor types correlates with specific clinical pathological characteristics, malignant potential and poor prognosis (25). Some authors have observed the most prominent Glut1 expression around the necrotic areas or the hypoxic regions of tumors $(26,27)$. Although cancer glucose uptake is thought to be primarily driven by Glut1, recently it was shown that Glut3, but not Glut1, correlates with poor survival of patients with brain tumors and other cancers (28). Notably, mRNA of some other glucose transporters were changed in tumors of our patients, e.g. Glut2 was markedly downregulated in 10 tumors (fold of decrease $9.0 \pm 1.5$; Fig. 2).

Glucosemetabolismandelectron transportchain.Cancercells metabolize glucose mostly via glycolysis, even in the presence of sufficient oxygen (5). Therefore, in our patients we focused primarily on pathways producing energy. We observed that glycolytic pathway is affected very significantly in ccRCCs $\left(\mathrm{P}=2.5 \times 10^{8}\right.$; Table I). Hexokinase 2 (HK2) and 3 (HK3) were upregulated in ccRCCs. From 11 patients, gene expression of the HK 2 in tumors was increased in 9 patients (fold of increase $34.2 \pm 7.5$; Fig. 2), in the patient with renal oncocytoma it was downregulated and in $1 \mathrm{ccRCC}$ patient it was not changed. 

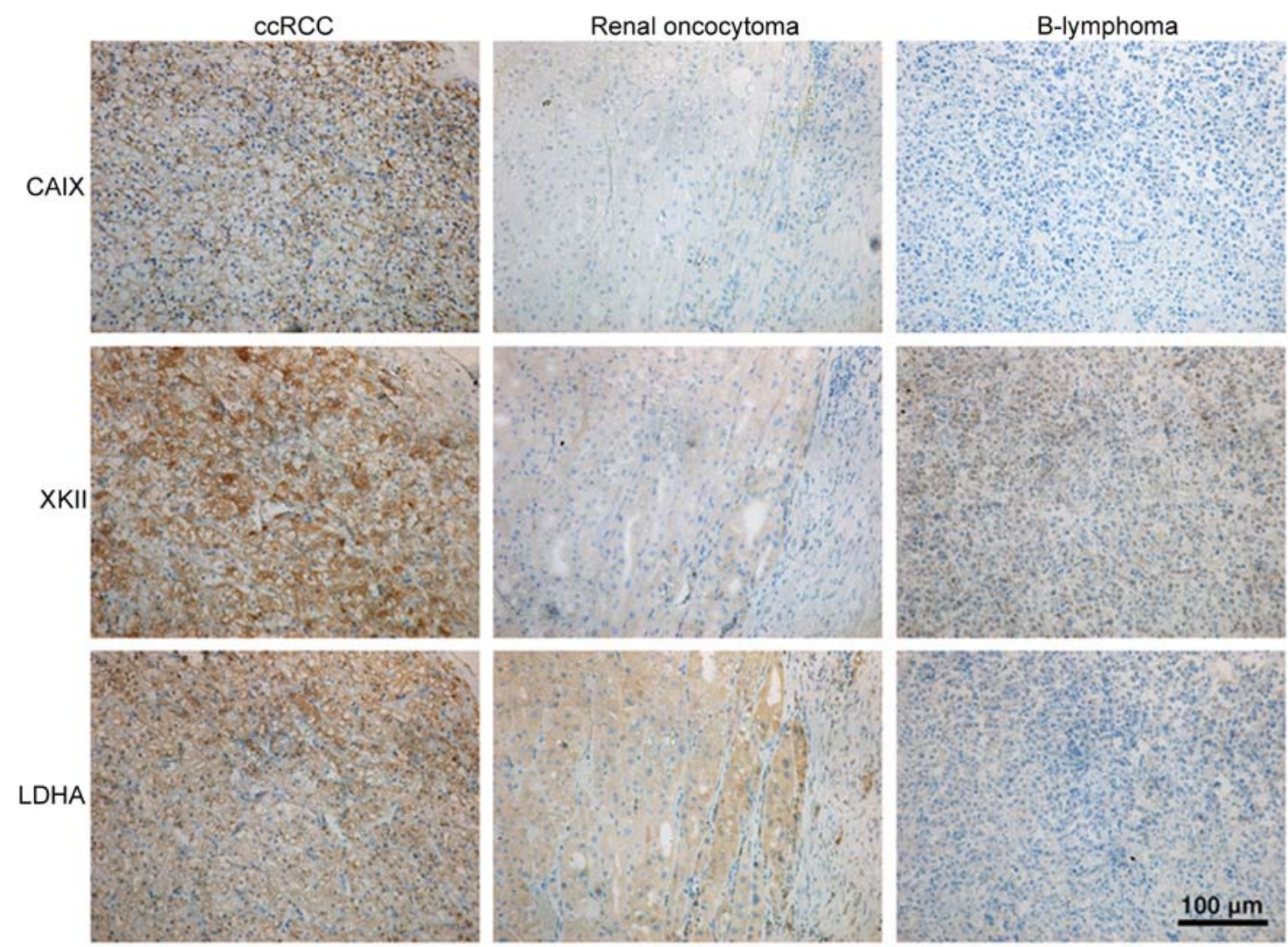

Figure 3. Immunohistochemical analysis of serial tissue sections from three different tissue specimens. Sections (4 $\mu \mathrm{m})$ from clear cell renal cell carcinoma, oncocytoma and B-lymphoma were stained for carbonic anhydrase IX (CAIX), hexokinase II (HKII) and lactate dehydrogenase A (LDHA). Strong CAIXrelated staining signal was predominantly localized in the plasma membranes of ccRCC cells, but was completely absent in the oncocytoma and B-lymphoma sections. Specific HKII and LDHA staining pattern was observed in all tissue specimens, with ccRCC sample the most strongly positive.

Immunohistochemistry also proved the robust HK2 signal in ccRCC tumors, weak signal in B-lymphoma and almost no signal in renal oncocytoma (Fig. 3). HK3 mRNA was elevated

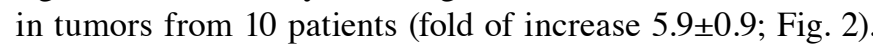
HK2, a pivotal glycolytic enzyme is often overexpressed in tumor cells and contributes to glycolysis. Recently, it was found that HK2 protein is increased in cancer-associated fibroblasts (29). Also, current studies demonstrated that HK2 is overexpressed and promotes glycolysis in tumor cells, but not in normal cells $(30,31)$. Moreover, HK2 overexpression is associated with a short progression-free survival, which could be associated with chemoresistance of the epithelial ovarian cancer (32). Conversion of the fructose 6-phosphate to fructose-1,6-bisphosphate is catalyzed by phosphofructokinase (PFK). We observed significant increase in $\mathrm{P}$ isoform of the PFK (PFKP), but not in L or M isoforms (Fig. 2). PFKP was shown to play a critical role in cell proliferation in breast cancer cells. It is suggested that a transcription factor KLF4 plays a role in the maintenance of high glycolytic metabolism by transcriptional activation of the PFKP gene in breast cancer cells (33). Phosphoglycerate mutase (PGAM) of type 1 was $>2$-fold increased, but that of type 2 (PGAM2) was decreased 7.2 \pm 1.3 -fold in 7 out of 11 patients (Fig. 2). Recently, it was shown that gluconeogenic enzyme fructose1,6-bisphosphatase 1 (FBP1) was uniformly depleted in over 600 ccRCC tumors examined (34). The human FBP1 locus resides on chromosome $9 \mathrm{q} 22$, the loss of which is associated with poor prognosis for ccRCC patients. In our patients, we observed strong decrease of the FBP1 in the ccRCC tumors (13.9 \pm 2.5 -fold in 9 from 11 patients), which is in line with the results of $\mathrm{Li}$ and co-workers (34). While the glycolytic pathway in these tumors is upregulated, gene expression of proteins involved in the gluconeogenesis is suppressed, except of aldolase C (ALDOC), which was significantly increased in 8 out of 9 ccRCC patients (Fig. 2). Pyruvate is the end product of cytosolic glycolysis and has a variety of possible fates. In tumors, majority of the pyruvate is converted to lactate by lactate dehydrogenase (LDH) of type A (LDHA), which was increased 5.7 \pm 0.8 -fold in 10 tumors. Immunohistochemical staining revealed strong LDHA signal in ccRCC tumor, weak signal in renal oncocytoma and no signal in B-lymphoma (Fig. 3). LDHA upregulation was observed also in the study of Girgis and co-workers (35) on the 170 ccRCC samples. Thus, upregulation of the LDHA could be a predictor of poor prognosis in clear cell renal cell carcinoma. On the other hand, gene expression of the LDHB was significantly suppressed in 8 tumors compared to corresponding healthy tissues (3.5 \pm 0.4 -fold). Notably, the patient with renal oncocytoma had significantly increased expression of LDHB (Fig. 2, dark column). In human cancers, overexpressed LDHA was associated with the increased aggressiveness (36).

In healthy cells majority of the pyruvate is used for the mitochondrial oxidation. One of the most consistent hallmarks of cancer biology is the preference of tumor cells to derive energy through glycolysis as opposed to the more efficient process of oxidative phosphorylation (OXPHOS). By 
A
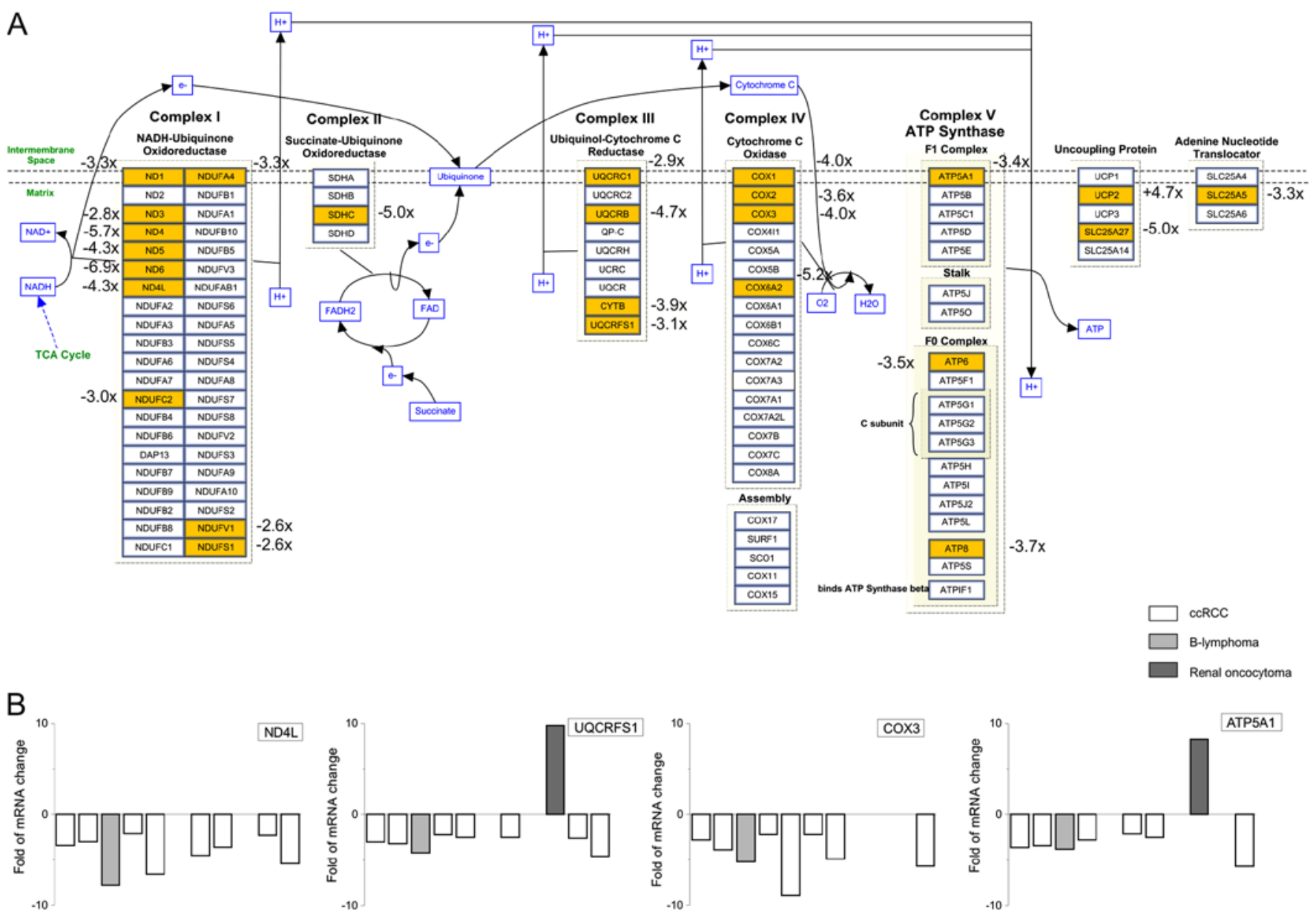

Figure 4. Mitochondrial electron transport chain. (A) Genes of several enzymes of the complexes I-V in the mitochondrial respiratory chain were decreased in ccRCCs, but not in the patient with renal oncocytoma. (B) Expression of the genes coding for ND4L, UQCRFS1, COX3 and ATP5A1 in individual patients with ccRCC (empty columns), B-lymphoma (light gray column) and renal oncocytoma (dark gray column).

controlling the mitochondrial flow of pyruvate, a cancer cell can tune its physiology to meet the demands of rapid growth (37). Mitochondrial pyruvate carrier of type 1 (MPC1), but not 2 (MPC2) was slightly decreased in tumors, compared to the matched control tissue. Since pyruvate transport is a rate-limiting step in pyruvate oxidation, downregulation of the MPC1 can dampen its further utilization, e.g. electron transport chain. Indeed, the gene expression of several proteins involved in electron transport chain was significantly decreased $(\mathrm{P}<0.0044$; Table I).

In ccRCC tumors, we observed decrease in the gene expression of proteins in complex I, II, III, IV and V (Fig. 4). Mayr et al (38) showed that loss of respiratory chain complex I (NADH/ubiquinone oxidoreductase) is associated with renal oncocytoma. In ccRCC tumors, we observed rapid decrease in NADH dehydrogenase (ND) type 1, 3, 4, 5 and 4L mRNA compared to healthy part of the tissue (Fig. 4). Cytochrome $c$ oxidase (COX) is a crucial enzyme of the complex IV. We observed decrease in the mRNA levels of COX1, COX2 and COX3, with the highest decrease in the COX3 levels (3.9 \pm 0.9 -fold; $n=7$ ccRCC tumors; Fig. 4).

In complex V, ATP5A1 was significantly downregulated in ccRCC tumors. This observation is in agreement with the work of Yusenko and co-workers (39), who observed downregulation of ATP5A1, the $\alpha$ subunit of complex V in chromophobe
RCCs. In majority of our patients with ccRCC, ATP5A1 was also significantly decreased (fold-decrease $2.5 \pm 0.7 ; n=6$ ccRCC patients; Fig. 4).

Cluster analysis. Cluster analysis based on the volume difference of tumor/healthy tissue pairs was performed using selected set of genes consisting of all significantly changed genes involved in glycolysis and hypoxia-related metabolic pathways (Fig. 5). Most of the ccRCC samples were clustered together with a highly similar gene expression pattern, except one ccRCC sample, which was more variable. However, oncocytoma sample differs from other samples forming a separate subgroup in the cluster tree. Gene clustering into two main subgroups is based on their upregulation or downregulation in individual samples: all genes of the electron transport chain were clustered together due to downregulation of the whole pathway.

Cancer cells reprogram their metabolism in order to satisfy their bioenergetic and biosynthetic requirements. These cells display reduced ability to use mitochondrial oxidation and favor the conversion of pyruvate into lactate, despite the availability of oxygen. Overproduction of the lactate activates $\mathrm{pH}$ regulating transport systems, including CAIX as its catalytic component. Beroukhim and co-workers (11) suggested that the assessment of appropriate markers of the VHL pathway 


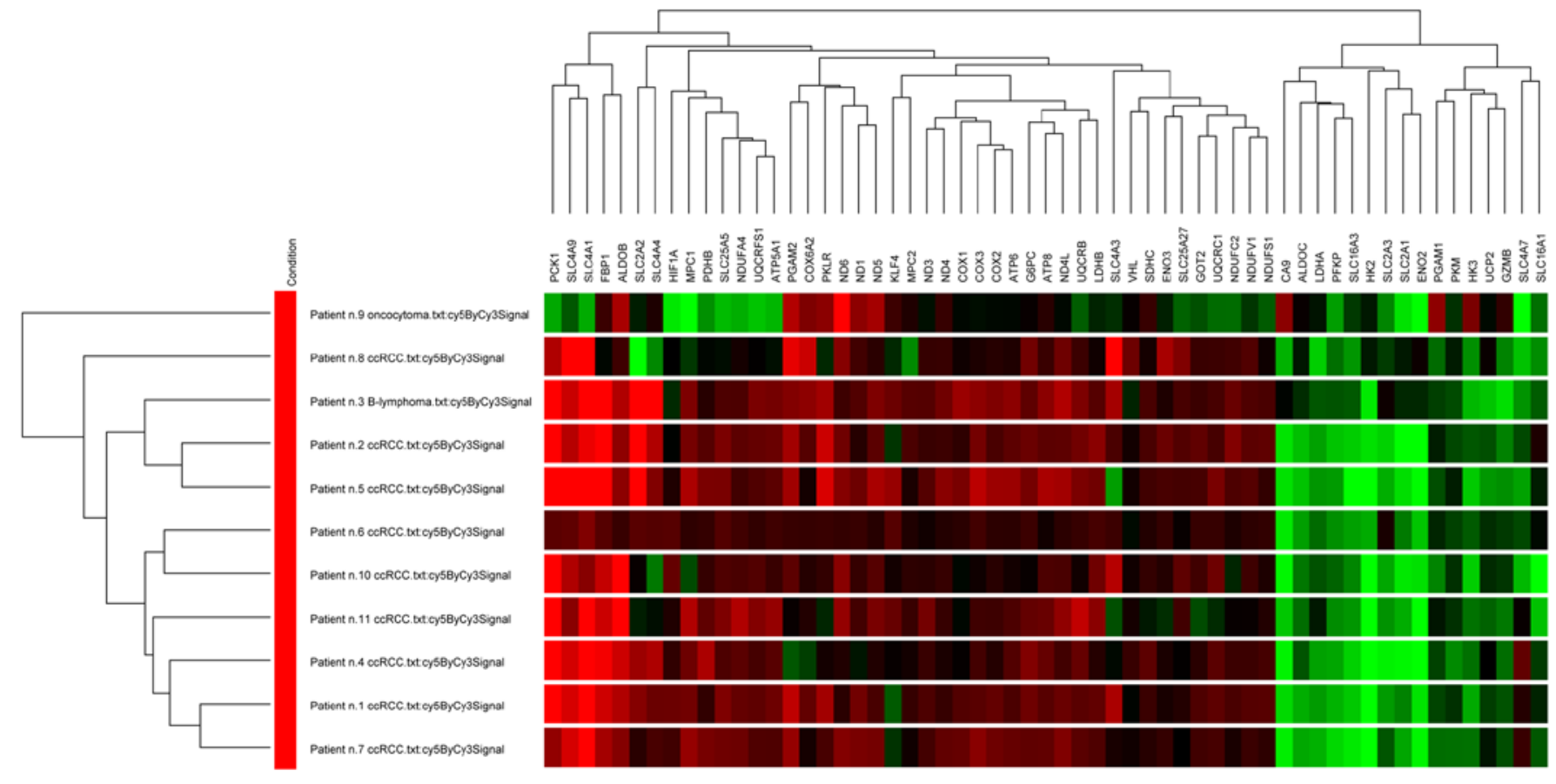

Figure 5. Hierarchical clustering analysis of the genes with significantly altered expression between ccRCC and matching normal kidney tissue. Cluster analysis includes the genes involved in $\mathrm{pH}$ regulation and energy metabolism in ccRCCs. Representative cluster analysis was performed from 9 patients with ccRCC, 1 patient with B-lymphoma and 1 patient with renal oncocytoma. Gene expression was considered to be significantly changed (red, downregulated; green, upregulated), when the change between healthy tissue and ccRCC in most of samples was observed to be at least 2-times.

dysregulation (e.g., expression of gene coding for CA IX) may be more useful in predicting response to therapy than assessment of the VHL inactivation status.

To compensate the inefficient extraction of energy from glucose and to maintain the biomass production, malignant cells have an at least a 20- to 30-fold higher rate of glycolysis than normal cells (25). Mitochondrial impairment increases from the less aggressive to the most aggressive RCCs, and correlates with a considerably decreased content of OXPHOS complexes (complexes II, III, and IV of the respiratory chain, and ATPase/ATP synthase) rather than to the mitochondrial content (citrate synthase and mitochondrial (mt)DNA) (40). The present study is among the first showing complex changes in the glycolytic pathway and mitochondrial respiration in tumors of patients suffering from ccRCCs. Moreover, our results nicely correlate with other studies showing strong upregulation of CAIX (41), Glut1 (42), LDHA $(35,43)$ and downregulation of AE1, FBP1 (34) and all complexes of the mitochondrial respiratory chain in ccRCC. Also, these results correlate with changes observed in animal studies and/or cell cultures, e.g., distorted tubules in TRACK (transgenic model of cancer of the kidney) mice exhibit higher levels of CA IX, Glut1, and VEGF than tubules in non-transgenic control mice (44). Specific genes and signaling molecules involved in the tumor-forced glycolysis and related phenomena, such as $\mathrm{pH}$ regulation, may represent potential therapeutic targets of agents that specifically interact with the key factors of tumor phenotype. Although some groups $(45,46)$, dispute the role of CA IX as a prognostic marker of the ccRCC, we believe that together with Glut1, Glut3, HK2, FBP1, ALDOC and LDHA, CA IX is an important biomarker for ccRCC diagnosis. Moreover, targeting these proteins might be of therapeutic importance.

\section{Acknowledgements}

The present study was supported by grants APVV-0045-11, APVV-0108-10, VEGA 2/0074/13 and CEMAN.

\section{References}

1. Cheville JC, Lohse CM, Zincke H, Weaver AL and Blute ML: Comparisons of outcome and prognostic features among histologic subtypes of renal cell carcinoma. Am J Surg Pathol 27: 612-624, 2003.

2. Weiss RH and Lin PY: Kidney cancer: Identification of novel targets for therapy. Kidney Int 69: 224-232, 2006.

3. Bonassi S and Neri M: Genetic biomarkers in human population studies. In: Handbook of Genomic Medicine. Willard HF and Ginsburg GS (eds). Elsevier, 2008.

4. Beck SD, Patel MI, Snyder ME, Kattan MW, Motzer RJ, Reuter VE and Russo P: Effect of papillary and chromophobe cell type on disease-free survival after nephrectomy for renal cell carcinoma. Ann Surg Oncol 11: 71-77, 2004.

5. Warburg O: On respiratory impairment in cancer cells. Science 124: 269-270, 1956.

6. Matsumoto S, Hyodo F, Subramanian S, Devasahayam N, Munasinghe J, Hyodo E, Gadisetti C, Cook JA, Mitchell JB and Krishna MC: Low-field paramagnetic resonance imaging of tumor oxygenation and glycolytic activity in mice. J Clin Invest 118: 1965-1973, 2008.

7. Vaupel P: Metabolic microenvironment of tumor cells: A key factor in malignant progression. Exp Oncol 32: 125-127, 2010.

8. Wenger RH, Stiehl DP and Camenisch G: Integration of oxygen signaling at the consensus HRE. Sci STKE 2005: re12, 2005.

9. Trayhurn P, Wang B and Wood IS: Hypoxia and the endocrine and signalling role of white adipose tissue. Arch Physiol Biochem 114: 267-276, 2008.

10. Gnarra JR, Glenn GM, Latif F, Anglard P, Lerman MI, Zbar B and Linehan WM: Molecular genetic studies of sporadic and familial renal cell carcinoma. Urol Clin North Am 20: 207-216, 1993. 
11. Beroukhim R, Brunet JP, Di Napoli A, Mertz KD, Seeley A, Pires MM, Linhart D, Worrell RA, Moch H, Rubin MA, et al: Patterns of gene expression and copy-number alterations in Von Hippel-Lindau disease-associated and sporadic clear cell carcinoma of the kidney. Cancer Res 69: 4674-4681, 2009.

12. Baldewijns MM, van Vlodrop IJ, Vermeulen PB, Soetekouw PM, van Engeland M and de Bruïne AP: VHL and HIF signalling in renal cell carcinogenesis. J Pathol 221: 125-138, 2010.

13. Pinthus JH, Whelan KF, Gallino D, Lu JP and Rothschild N: Metabolic features of clear-cell renal cell carcinoma: Mechanisms and clinical implications. Can Urol Assoc J 5: 274-282, 2011.

14. Wykoff CC, Beasley NJ, Watson PH, Turner KJ, Pastorek J, Sibtain A, Wilson GD, Turley H, Talks KL, Maxwell PH, et al: Hypoxia-inducible expression of tumor-associated carbonic anhydrases. Cancer Res 60: 7075-7083, 2000.

15. Bui MH, Seligson D, Han KR, Pantuck AJ, Dorey FJ, Huang Y, Horvath S, Leibovich BC, Chopra S, Liao SY, et al: Carbonic anhydrase IX is an independent predictor of survival in advanced renal clear cell carcinoma: Implications for prognosis and therapy. Clin Cancer Res 9: 802-811, 2003.

16. Takacova M, Bartosova M, Skvarkova L, Zatovicova M, Vidlickova I, Csaderova L, Barathova M, Breza J Jr, Bujdak P, Pastorek J, et al: Carbonic anhydrase IX is a clinically significant tissue and serum biomarker associated with renal cell carcinoma. Oncol Lett 5: 191-197, 2013.

17. Sedlakova O, Svastova E, Takacova M, Kopacek J, Pastorek J and Pastorekova S: Carbonic anhydrase IX, a hypoxia-induced catalytic component of the $\mathrm{pH}$ regulating machinery in tumors. Front Physiol 4: 400, 2014

18. Ditte P, Dequiedt F, Svastova E, Hulikova A, OhradanovaRepic A, Zatovicova M, Csaderova L, Kopacek J, Supuran CT, Pastorekova S, et al: Phosphorylation of carbonic anhydrase IX controls its ability to mediate extracellular acidification in hypoxic tumors. Cancer Res 71: 7558-7567, 2011

19. Svastova E, Witarski W, Csaderova L, Kosik I, Skvarkova L, Hulikova A, Zatovicova M, Barathova M, Kopacek J, Pastorek J, et al: Carbonic anhydrase IX interacts with bicarbonate transporters in lamellipodia and increases cell migration via its catalytic domain. J Biol Chem 287: 3392-3402, 2012.

20. Gorbatenko A, Olesen CW, Boedtkjer E and Pedersen SF: Regulation and roles of bicarbonate transporters in cancer. Front Physiol: 16 April. 2014. doi: 10.3389/fphys.2014.00130.

21. Karumanchi SA, Jiang L, Knebelmann B, Stuart-Tilley AK, Alper SL and Sukhatme VP: VHL tumor suppressor regulates $\mathrm{Cl}^{-} / \mathrm{HCO}_{3}{ }^{-}$exchange and $\mathrm{Na}^{+} / \mathrm{H}^{+}$exchange activities in renal carcinoma cells. Physiol Genomics 5: 119-128, 2001.

22. Halestrap AP: The SLC16 gene family: structure, role and regulation in health and disease. Mol Aspects Med 34: 337-349, 2013

23. Doyen J, Trastour C, Ettore F, Peyrottes I, Toussant N, Gal J, Ilc K, Roux D, Parks SK, Ferrero JM, et al: Expression of the hypoxiainducible monocarboxylate transporter MCT4 is increased in triple negative breast cancer and correlates independently with clinical outcome. Biochem Biophys Res Commun 451: 54-61, 2014.

24. Pértega-Gomes N, Vizcaíno JR, Attig J, Jurmeister S, Lopes C and Baltazar F: A lactate shuttle system between tumour and stromal cells is associated with poor prognosis in prostate cancer. BMC Cancer 14: 352, 2014.

25. Jóźwiak P, Krześlak A, Pomorski L and Lipińska A: Expression of hypoxia-related glucose transporters GLUT1 and GLUT3 in benign, malignant and non-neoplastic thyroid lesions. Mol Med Rep 6: 601-606, 2012.

26. Brown RS, Goodman TM, Zasadny KR, Greenson JK and Wahl RL: Expression of hexokinase II and Glut-1 in untreated human breast cancer. Nucl Med Biol 29: 443-453, 2002

27. Mori Y, Tsukinoki K, Yasuda M, Miyazawa M, Kaneko A and Watanabe Y: Glucose transporter type 1 expression are associated with poor prognosis in patients with salivary gland tumors. Oral Oncol 43: 563-569, 2007

28. Flavahan WA, Wu Q, Hitomi M, Rahim N, Kim Y, Sloan AE, Weil RJ, Nakano I, Sarkaria JN, Stringer BW, et al: Brain tumor initiating cells adapt to restricted nutrition through preferential glucose uptake. Nat Neurosci 16: 1373-1382, 2013

29. Hu JW, Sun P, Zhang DX, Xiong WJ and Mi J: Hexokinase 2 regulates G1/S checkpoint through CDK2 in cancer-associated fibroblasts. Cell Signal 26: 2210-2216, 2014.
30. Feliciano A, Castellvi J, Artero-Castro A, Leal JA, Romagosa C, Hernández-Losa J, Peg V, Fabra A, Vidal F, Kondoh HC, et al: miR-125b acts as a tumor suppressor in breast tumorigenesis via its novel direct targets ENPEP, CK2- $\alpha, \mathrm{CCNJ}$, and MEGF9. PLoS One 8: e76247, 2013.

31. Patra KC, Wang Q, Bhaskar PT, Miller L, Wang Z, Wheaton W, Chandel N, Laakso M, Muller WJ, Allen EL, et al: Hexokinase 2 is required for tumor initiation and maintenance and its systemic deletion is therapeutic in mouse models of cancer. Cancer Cell 24: $213-228,2013$.

32. Suh DH, Kim MA, Kim H, Kim MK, Kim HS, Chung HH, Kim YB and Song YS: Association of overexpression of hexokinase II with chemoresistance in epithelial ovarian cancer. Clin Exp Med 14: 345-353, 2014

33. Moon JS, Kim HE, Koh E, Park SH, Jin WJ, Park BW, Park SW and Kim KS: Krüppel-like factor 4 (KLF4) activates the transcription of the gene for the platelet isoform of phosphofructokinase (PFKP) in breast cancer. J Biol Chem 286: 23808-23816, 2011.

34. Li B, Qiu B, Lee DS, Walton ZE, Ochocki JD, Mathew LK, Mancuso A, Gade TP, Keith B, Nissim I, et al: Fructose-1,6bisphosphatase opposes renal carcinoma progression. Nature 13: 251-255, 2014.

35. Girgis H, Masui O, White NM, Scorilas A, Rotondo F, Seivwright A, Gabril M, Filter ER, Girgis AH, Bjarnason GA, et al: Lactate dehydrogenase A is a potential prognostic marker in clear cell renal cell carcinoma. Mol Cancer 13: 101, 2014.

36. Xie H, Hanai J, Ren JG, Kats L, Burgess K, Bhargava P, Signoretti S, Billiard J, Duffy KJ, Grant A, et al: Targeting lactate dehydrogenase--a inhibits tumorigenesis and tumor progression in mouse models of lung cancer and impacts tumor-initiating cells. Cell Metab 19: 795-809, 2014.

37. Schell JC and Rutter J: The long and winding road to the mitochondrial pyruvate carrier. Cancer Metab 1: 6, 2013.

38. Mayr JA, Meierhofer D, Zimmermann F, Feichtinger R, Kögler C, Ratschek M, Schmeller N, Sperl W and Kofler B: Loss of complex I due to mitochondrial DNA mutations in renal oncocytoma. Clin Cancer Res 14: 2270-2275, 2008.

39. Yusenko MV, Ruppert T and Kovacs G: Analysis of differentially expressed mitochondrial proteins in chromophobe renal cell carcinomas and renal oncocytomas by 2-D gel electrophoresis. Int J Biol Sci 6: 213-224, 2010

40. Simonnet H, Alazard N,Pfeiffer K, Gallou C, Béroud C, Demont J, Bouvier R, Schägger $\mathrm{H}$ and Godinot $\mathrm{C}$ : Low mitochondrial respiratory chain content correlates with tumor aggressiveness in renal cell carcinoma. Carcinogenesis 23: 759-768, 2002

41. Choueiri TK, Cheng S, Qu AQ, Pastorek J, Atkins MB and Signoretti S: Carbonic anhydrase IX as a potential biomarker of efficacy in metastatic clear-cell renal cell carcinoma patients receiving sorafenib or placebo: Analysis from the treatment approaches in renal cancer global evaluation trial (TARGET). Urol Oncol 31: 1788-1793, 2013.

42. Page T, Hodgkinson AD, Ollerenshaw M, Hammonds JC and Demaine AG: Glucose transporter polymorphisms are associated with clear-cell renal carcinoma. Cancer Genet Cytogenet 163: $151-155,2005$.

43. White EA, Kenny HA and Lengyel E: Three-dimensional modeling of ovarian cancer. Adv Drug Deliv Rev 79-80: 184-192, 2014.

44. Fu L, Wang G, Shevchuk MM, Nanus DM and Gudas LJ: Generation of a mouse model of Von Hippel-Lindau kidney disease leading to renal cancers by expression of a constitutively active mutant of HIF1 $\alpha$. Cancer Res 71: 6848-6856, 2011.

45. Leibovich BC, Sheinin Y, Lohse CM, Thompson RH, Cheville JC, Zavada $\mathbf{J}$ and Kwon ED: Carbonic anhydrase IX is not an independent predictor of outcome for patients with clear cell renal cell carcinoma. J Clin Oncol 25: 4757-4764, 2007.

46. Zhang BY, Thompson RH, Lohse CM, Dronca RS, Cheville JC, Kwon ED and Leibovich BC: Carbonic anhydrase IX (CAIX) is not an independent predictor of outcome in patients with clear cell renal cell carcinoma (ccRCC) after long-term follow-up. BJU Int 111: 1046-1053, 2013. 